\title{
Padrões alimentares de mulheres no climatério em atendimento ambulatorial no Sul do Brasil
}

\author{
Dietary patterns in menopausal women \\ receiving outpatient care in Southern Brazil
}

Maichelei Hoffmann ${ }^{1}$

Karina Giane Mendes ${ }^{2}$

Raquel Canuto ${ }^{3}$

Anderson da Silva Garcez ${ }^{1}$

Heloísa Theodoro ${ }^{2}$

Alice Dalpicolli Rodrigues ${ }^{1}$

Maria Teresa Anselmo Olinto ${ }^{1}$

Programa de PósGraduação em Saúde Coletiva, Universidade do Vale do Rio dos Sinos. Av. Unisinos 950, Cristo Rei. 93022-000 São Leopoldo RS Brasil.mtolinto@gmail.com ${ }^{2}$ Centro de Ciências Biológicas e da Saúde, Universidade de Caxias do Sul.

${ }^{3}$ Departamento de Nutrição, Universidade Federal do Rio Grande do Sul.

\begin{abstract}
The scope of this paper is to identify dietary patterns and the relationship with menopausal status. It involved a cross-sectional study with 646 women in outpatient care in southern Brazil. Dietary intake was assessed by a frequency questionnaire and five dietary patterns were identified by main component analysis. The menopausal status was classified as premenopausal, perimenopausal and postmenopausal women. Five dietary patterns were identified: fruit and vegetables; Brazilian fare (rice, beans and milk); snacks (cake, burgers, pizza and sweetbread); health diet (fish, fruit juice, bread and vegetable soup), and regional (typical food in the Serra Gaucha, like red meat, pasta and yams). After adjusting for sociodemographic characteristics, there was no significant association between menopausal status and dietary patterns. Only age, education and income were associated with dietary patterns. Five dietary patterns that described the food consumption of the population studied were identified, which are similar to those indicated as ideal for the Brazilian population. The findings reveal that the dietary pattern of women in menopause are significantly influenced by age, education and income but are not influenced by the menopausal status per se.
\end{abstract}

Key words Dietary patterns, Menopause, Women, Factor analysis
Resumo Objetivo: Identificar os padrões alimentares e sua relação com o estado menopáusico. Métodos: Estudo transversal com 646 mulheres em atendimento ambulatorial no Sul do Brasil. O consumo alimentar foi avaliado por questionário de frequência e cinco padrões alimentares foram identificados através da análise de componentes principais. O estado menopáusico foi classificado em pré-menopausa, perimenopausa e pós-menopausa. Resultados: Identificaram-se cinco padrões alimentares: frutas e verduras, brasileiro (arroz, feijão e leite), lanches (bolo, xis, pizza, cuca), prudente (peixe, suco natural, pão integral e sopa de legumes) e regional (alimentos típicos na serra gaúcha como carnes vermelhas, aipim e massas). Após ajuste para características sociodemográficas, não verificou-se associação significativa entre o estado menopáusico e os padrões alimentares. Apenas idade, escolaridade e renda mostraram-se associadas com padrões alimentares. Conclusão: Foram identificados cinco padrões alimentares que descreveram o consumo alimentar da população estudada, sendo estes semelhantes ao indicado como ideal para a população brasileira. Nossos achados indicam que o padrão alimentar das mulheres no climatério possui influência significativa da idade, escolaridade e renda e que não sofre influência do estado menopáusico.

Palavras-chave Padrão alimentar, Climatério, Mulheres, Análise fatorial 


\section{Introdução}

O aumento da expectativa de vida, o papel social e a maior inserção da mulher no mercado de trabalho demandam que o climatério, ocorrendo em plena vida produtiva, mereça maior interesse de pesquisadores da área da saúde da mulher. $\mathrm{O}$ climatério representa a transição gradual da fase reprodutiva para a não reprodutiva da mulher, iniciando aos 40 e terminando aos 65 anos de idade, e é dividido em três períodos: pré-menopausa, perimenopausa e pós-menopausa ${ }^{1}$. Neste período ocorrem inúmeras mudanças biológicas, endócrinas e clínicas devido às alterações hormonais, as quais geram diversos sintomas que implicam na qualidade de vida, bem como tornam as mulheres mais vulneráveis a certos agravos à saúde².

Dentre as principais disfunções orgânicas que podem ocorrer durante o período de climatério estão as alterações do metabolismo ósseo, facilitando a ocorrência da osteoporose; no metabolismo lipídico, com o aumento da lipoproteína de baixa densidade e diminuição da de alta densidade, tornando maior o risco de doenças cardiovasculares; e a substituição de musculatura por tecido adiposo acarretando em maior propensão ao ganho de peso ${ }^{3-8}$. Além disso, um fator que apresenta uma ação decisiva no estabelecimento dessas alterações é a alimentação, tornando-se de suma importância sua avaliação. Esta pode ser analisada por meio da identificação de padrões alimentares, que representam um retrato geral do consumo de alimentos e de nutrientes, caracterizados com base no hábito de ingestão usual ${ }^{9}$.

Estudos sobre o padrão alimentar de mulheres no climatério vêm assumindo expressiva importância, uma vez que esta fase compreende um período relativamente longo da vida da mulher e a alimentação adotada possui impacto sobre sua saúde. Além disso, a literatura científica tem demonstrado a relação de determinados padrões alimentares com a saúde do indivíduo, tanto como fator de proteção quanto de risco para comorbidades ${ }^{4,10,11}$.

Dessa forma, o presente estudo tem como objetivo identificar os padrões alimentares de mulheres atendidas em ambulatório de climatério do Sul do Brasil e as relações com o estado menopáusico. O conhecimento dos hábitos alimentares das mulheres nesta fase pode ter importantes implicações para saúde pública no correto direcionamento das ações de prevenção e tratamento de doenças, pricipalmente na formulação de orientações e intervenções de educação nutricio- nal, visando contribuir para proposição de medidas que amenizem os agravos deste período?.

\section{Métodos}

\section{População de Estudo}

Trata- se de um estudo transversal, com amostra de mulheres que frequentaram os ambulatórios de Climatério e Cirurgia Ginecológica do Sistema Único de Saúde de Caxias do Sul (RS), entre janeiro de 2010 e maio de 2011. Esta investigação faz parte de uma pesquisa intitulada "Síndrome metabólica e estado menopausal em mulheres atendidas em um ambulatório de climatério no sul do Brasil". Outros achados e mais informações sobre a pesquisa podem ser encontrados em publicações prévias ${ }^{12,13}$.

As mulheres foram selecionadas na sala de espera do ambulatório, onde todas as pacientes foram convidadas a responder a entrevista e as pacientes que preenchiam os critérios de elegibilidade foram incluídas no estudo. O percentual de perdas por negativa de resposta ao questionário foi de $6 \%$. A amostra foi constituída de mulheres entre 40 e 65 anos, não histerectomizadas, que não foram submetidas à oforectomia bilateral e que não utilizavam terapia de reposição hormonal.

A presente pesquisa foi aprovada pelo Comitê de Ética em Pesquisa da Universidade de Caxias do Sul. O Termo de Consentimento Livre e Esclarecido foi assinado por todas as participantes.

\section{Coleta de dados}

As informações sobre o estado menopáusico e as características demográficas, socioeconômicas, antropométricas e de consumo alimentar foram obtidas por meio de questionários padronizados, pré-codificados e pré-testados, aplicados por entrevistadoras previamente treinadas. O estado menopáusico foi classificado em: pré-menopausa, sendo incluídas as mulheres que relataram apresentar ciclos menstruais regulares em relação à periodicidade e fluxo; perimenopausa, aquelas que possuíam ciclos irregulares, e pós-menopausa, as que não apresentavam ciclos menstruais há mais de 12 meses $^{1}$.

As características demográficas avaliadas e categorizadas foram: idade (40 a 45 anos; 46 a 50 anos; 51 a 55 anos; 56 a 65 anos), cor da pele autorreferida (branca e não branca) e estado civil (casada/em união estável e não casada). As característi- 
cas socioeconômicas avaliadas foram: escolaridade em anos completos de estudo $(\geq 12 ; 9$ a $11 ; 5$ a $8 \mathrm{e} \leq$ 4 anos) e renda familiar em salários mínimos considerando o valor da época de $\mathrm{R} \$ 510,00(\geq 5,01$; 3,01 a 5,00; 2,01 a 3,00 e $\leq 2$ salários).

Para aferição do peso corporal foi utilizada a balança antropométrica de travessão (marca Welmy) com pesos móveis, devidamente calibrada, com capacidade de $150 \mathrm{~kg}$ e precisão de $100 \mathrm{~g}$. As mulheres foram pesadas na posição em pé, descalças, com o mínimo de roupa possível e com os braços estendidos ao lado do corpo. A verificação da altura foi realizada no estadiômetro, com capacidade de $2 \mathrm{~m}$ e precisão de 1 $\mathrm{mm}$, acoplado à balança antropométrica descrita anteriormente ${ }^{14}$. Para aferição, as mulheres ficavam na posição em pé, descalças, com os braços estendidos ao lado do corpo e a cabeça reta. As medidas de peso e altura foram realizadas uma única vez. O estado nutricional foi avaliado pelo Índice de Massa Corporal (IMC), razão entre o peso $(\mathrm{kg})$ e o quadrado da altura $(\mathrm{m})$, sendo classificado como normal $\left(\leq 24,9 \mathrm{~kg} / \mathrm{m}^{2}\right)$, sobrepeso $\left(25\right.$ a $\left.29,9 \mathrm{~kg} / \mathrm{m}^{2}\right)$ e obeso $\left(\geq 30 \mathrm{~kg} / \mathrm{m}^{2}\right)^{15}$.

\section{Avaliação do consumo alimentar}

Para obter as informações sobre o consumo alimentar foi utilizado um Questionário de Frequência Alimentar Qualitativo (QFA) adaptado do estudo de Anjos et al. ${ }^{16}$ para contemplar os hábitos alimentares das mulheres no climatério da região de realização do estudo. O QFA foi composto por 74 alimentos ou itens alimentares investigados quanto ao número de vezes (de zero a sete vezes) de consumo ao dia ou semana ou mês ou ano ${ }^{16}$. O consumo dos itens alimentares foi transformado em frequência de consumo anual e para os alimentos com consumo apenas na época foi feita ponderação (frequência de consumo dividido por quatro estações do ano).

Para obtenção dos padrões alimentares utilizou-se análise fatorial exploratória e para extração dos fatores o método de análise de componentes principais. Primeiramente, foram formados 46 grupos alimentares a partir dos 74 itens alimentares do QFA (Quadro 1), tendo como critérios: composição nutricional, correlação significativa entre os itens $(>0,20 ; p \leq 0,05)$, além de aspectos culturais. Devido a resultados não satisfatórios, foram excluídos os itens com consumo abaixo de $20 \%$ e os que não eram considerados alimentos (Quadro 1). Para avaliar a pertinência da análise fatorial, o coeficiente Kaiser-Mayer -Olkin (referência: $\mathrm{KMO} \geq 0,6$ ) e o teste de esfe-

Quadro 1. Grupos alimentares formados e alimentos excluídos.

\section{Grupos Alimentares}

\section{Arroz branco}

Batata cozida, assada, purê

Batata frita ou palha

Aipim, inhame

Macarrão, massas

Farofa

Feijão preto

Pão integral

Salgado frito, assado, biscoito

Carne de gado assada, cozida, grelhada

Ovo frito, cozido

Frango cozido, grelhado, assado

Carne de porco

Fígado de boi

Xis, pizza

Salsicha, presunto, mortadela, linguiça, salame,

salsichão

Leite integral

Refrigerante normal, refresco em pó

Suco natural

Banana

Bergamota, uva, mamão, melancia, manga

Maçã

Laranja

Sorvete

Alface, agrião, espinafre, rúcula, couve

Brócolis, couve flor, repolho

Chuchu, abobrinha, berinjela

Moranga, cenoura, beterraba, tomate

Sopa de legumes

Chimia: goiabada, figada, mel, marmelada

Bife de gado frito, frango frito, empanado

Peixe frito, ensopado, grelhado

Cuca, pão caseiro, bolo

Biscoito recheado e biscoito doce

Pão de forma/leite, pão sovadinho/francês

Todos os queijos

Margarina

Manteiga

\section{Alimentos Excluídos}

\section{Arroz integral}

Carne seca

Leite semi e desnatado

Leite de soja

Refrigerante zero

Açúcar refinado

Chimarrão

Chocolate e achocolatado

Adoçante 
ricidade de Bartlett (referência: $\mathrm{p} \leq 0,05$ ) foram aplicados. Rotação ortogonal (varimax) foi realizada para examinar a estrutura do padrão. O número de fatores a extrair foi definido observando os resultados referentes à variância total explicada e também pelo gráfico de Cattel (scree plot).

\section{Análise estatística}

Posterior à obtenção dos padrões alimentares, os respectivos escores foram divididos em tercis; sendo categorizados em baixo consumo ( $1^{\circ}$ e $2^{\circ}$ tercil) e alto consumo (tercil superior). Para investigar a associação entre cada um dos padrões identificados e as características da amostra foi empregado o teste de Qui-quadrado de Pearson. Os padrões alimentares que apresentaram associações estatisticamente significativas ( $\mathrm{p}$-valor $\leq 0,05)$ com estado menopáusico foram levados para análise multivariada. Foi utilizado o teste de Regressão de Poisson, com variância robusta, para o cálculo das razões de prevalência e seus respectivos intervalos de confiança de $95 \%$. As variáveis que apresentaram nível de significância menor que $20 \%$, na análise bruta, foram incluídas na análise multivariada que seguiu o modelo de análise, no qual no primeiro nível foram incluídas as variáveis sociodemográficas e no segundo nível (proximal) foram incluídas as variáveis do primeiro nível (sociodemográficas) que apresentaram $\mathrm{p} \leq 0,20$ e a variável estado nutricional. As análises foram realizadas nos Programas SPSS (Statistical Package for Social Sciences) versão 18.0 e STATA versão 12.0 (Stata Corp., College Station, Estados Unidos).

\section{Resultados}

Do total de 660 mulheres que atenderam aos critérios de inclusão e participaram do estudo, 14 foram excluídas por não apresentaram informações sobre padrões alimentares. Assim, a amostra final deste estudo compreendeu 646 mulheres. Diante do tamanho de amostra obtido, verificou-se um poder estatístico de $80 \%$ para detectar uma razão de prevalência de 1,35 entre estado menopáusico e padrões alimentares, adotandose um nível de confiança de $95 \%$ e uma frequência estimada de exposição (padrões alimentares) no grupo controle (pré-menopáusicas) de 35\%. Para identificar os padrões alimentares por meio de análise de componentes principais o tamanho de amostra necessário foi de 370 mulheres, segundo regra proposta por Hair ${ }^{17}$.
Foram identificados cinco padrões alimentares com $29,18 \%$ da variância explicada. O padrão alimentar I foi denominado frutas e verduras; o padrão alimentar II, formado por alimentos da dieta típica do brasileiro, tais como arroz, feijão e leite, foi denominado brasileiro; o padrão III foi denominado lanches por conter alimentos como xis, pizza, cuca, bolo; o padrão alimentar IV foi denominado prudente por conter alimentos tais como suco natural, peixe, pão integral, sopa de legumes entre outros; e o padrão alimentar V foi denominado regional por conter alimentos típicos da alimentação da serra gaúcha, composto por massa, aipim, carnes. O padrão que melhor representaria o consumo dessa população foi frutas e verduras, explicando $8,43 \%$ da variância (Tabela 1).

Na Tabela 2 são apresentadas as características sociodemográficas e do estado nutricional e menopáusico da amostra e a distribuição de elevado consumo (tercil superior) dos padrões alimentares. A maioria das mulheres era casada, tinha cor da pele branca, apresentava escolaridade menor que sete anos e renda familiar inferior a três salários mínimos. Dentre a amostra total, $80 \%$ estavam com sobrepeso ou obesidade e $46,5 \%$ das mulheres estavam na perimenopausa. $\mathrm{O}$ padrão alimentar frutas e verduras apresentou associação significativa com idade, estado nutricional e menopáusico, em que as mulheres que apresentavam maior consumo eram as com maior idade, obesas e menopausadas. O padrão alimentar tradicional mostrou associação significativa com escolaridade, sendo este mais consumido pelas mulheres com escolaridade média. O padrão alimentar lanches associou-se com estado nutricional e cor da pele, onde as mulheres brancas e com peso normal apresentaram maior consumo deste padrão. O padrão alimentar prudente associou-se significativamente com idade, sendo menos consumido pelas mulheres mais jovens; e o padrão alimentar regional com escolaridade, renda e estado menopáusico, sendo mais consumido pelas mulheres com baixa escolaridade e renda e que estavam na perimenopausa (Tabela 2).

Após análise multivariável, evidenciou-se associação direta entre maior consumo do padrão alimentar frutas e verduras com idade e uma associação inversa do padrão alimentar regional com escolaridade e renda das mulheres. Não foi verificada uma associação significativa entre o consumo dos padrões alimentares e o estado menopáusico (Tabela 3 ). 
PA II

Tabela 1. Padrões alimentares (PA) com os itens que o compõem, carga fatorial e \% de variância explicada.

\begin{tabular}{|c|c|c|c|}
\hline $\begin{array}{c}\text { Padrões } \\
\text { Alimentares }\end{array}$ & Itens alimentares & $\begin{array}{l}\text { Carga } \\
\text { fatorial }\end{array}$ & $\begin{array}{c}\% \text { da variância } \\
\text { explicada }^{*}\end{array}$ \\
\hline \multirow[t]{5}{*}{ PA I } & Brócolis, couve flor, repolho & 0,775 & 8,429 \\
\hline & Alface, agrião, espinafre, rúcula, couve & 0,668 & \\
\hline & Chuchu, berinjela, suqueti & 0,623 & \\
\hline & Moranga, cenoura, beterraba, tomate & 0,544 & \\
\hline & Bergamota, uva, mamão, melancia, manga & 0,501 & \\
\hline \multirow[t]{7}{*}{ PA II } & & & 6,931 \\
\hline & Feijão preto & 0,619 & \\
\hline & Manteiga & 0,611 & \\
\hline & Arroz branco & 0,608 & \\
\hline & Refrigerante, refresco em pó & 0,603 & \\
\hline & Laranja & 0,568 & \\
\hline & Leite integral & 0,445 & \\
\hline \multirow[t]{8}{*}{ PA III } & & & 5,442 \\
\hline & Queijos & 0,588 & \\
\hline & Embutidos & 0,584 & \\
\hline & Xis, pizza & 0,418 & \\
\hline & Cuca, bolo & 0,402 & \\
\hline & Pães (padaria) & 0,390 & \\
\hline & Chimia & 0,337 & \\
\hline & Biscoito doce, biscoito recheado & 0,323 & \\
\hline \multirow[t]{9}{*}{ PA IV } & & & 4,285 \\
\hline & Suco natural & 0,556 & \\
\hline & Salgado assado, frito, biscoito salgado & 0,489 & \\
\hline & Peixe & 0,476 & \\
\hline & Frango grelhado, assado, cozido & 0,427 & \\
\hline & Batata cozida, assada, purê & 0,421 & \\
\hline & Pão integral & 0,381 & \\
\hline & Sopa legumes & 0,377 & \\
\hline & Farofa & 0,324 & \\
\hline \multirow[t]{8}{*}{ PA V } & & & 4,091 \\
\hline & Carne de porco & 0,476 & \\
\hline & Bife de gado frito, frango frito, empanado & 0,465 & \\
\hline & Macarrão, massas & 0,456 & \\
\hline & Batata frita/palha & 0,427 & \\
\hline & Carne de gado assada, grelhada, ensopada & 0,425 & \\
\hline & Ovo frito, cozido & 0,425 & \\
\hline & Aipim, inhame & 0,324 & \\
\hline \multicolumn{2}{|c|}{ Total da variância explicada } & & $29,18 \%$ \\
\hline
\end{tabular}

PA I: frutas/verduras; PA II: brasileiro; PA III: lanches; PA IV: prudente; PA V: regional. ${ }^{*}$ KMO: 0,688; Esferacidade de Bartlet: $\leq 0,001$.

\section{Discussão}

No presente estudo foram descritos os padrões alimentares de mulheres no climatério em atendimento ambulatorial, levando em consideração o estado menopáusico como fator interferente no consumo dos padrões alimentares. $\mathrm{Na}$ análise ajustada, não houve associação com estado menopáusico, apenas as variáveis idade, escola- ridade e renda permaneceram associadas significativamente ao consumo de padrões alimentares.

Ao analisarmos os padrões podemos perceber que o frutas e verduras e o prudente foram compostos por alimentos considerados saudáveis, ou seja, um estilo mais benéfico de alimentação. Já o padrão lanches, formado por alimentos mais calóricos, ricos em proteínas, gorduras, carboidratos e sódio, apresentou uma dieta característica 
Tabela 2. Características da amostra e distribuição de elevado consumo (tercil superior) dos padrões alimentares (PA), segundo variáveis sociodemográficas e do estado nutricional e menopáusico de mulheres no climatério, em atendimento ambulatorial no Sul do Brasil $(\mathrm{N}=646)$.

\begin{tabular}{|c|c|c|c|c|c|c|}
\hline \multirow[b]{2}{*}{ Variável } & \multirow[b]{2}{*}{$\mathrm{N}(\%)$} & \multicolumn{5}{|c|}{ Padrões alimentares - elevado consumo } \\
\hline & & $\begin{array}{c}\text { PA I } \\
\text { (p-valor) } \\
\%\end{array}$ & $\begin{array}{c}\text { PA II } \\
\text { (p-valor) } \\
\%\end{array}$ & $\begin{array}{c}\text { PA III } \\
\text { (p-valor) } \\
\%\end{array}$ & $\begin{array}{c}\text { PA IV } \\
\text { (p-valor) } \\
\% \\
\end{array}$ & $\begin{array}{c}\text { PA V } \\
\text { (p-valor) } \\
\%\end{array}$ \\
\hline Idade & & $(0,001)^{*}$ & $(0,764)$ & $(0,099)$ & $(0,050)^{*}$ & $(0,248)$ \\
\hline 40 a 45 anos & $153(23,7)$ & 28,1 & 32,7 & 34,6 & 24,8 & 38,6 \\
\hline 46 a 50 anos & $182(28,2)$ & 28,6 & 35,7 & 39,6 & 37,9 & 35,2 \\
\hline 51 a 55 anos & $155(24,0)$ & 31,6 & 30,3 & 30,3 & 32,2 & 29,7 \\
\hline 56 a 65 anos & $156(24,1)$ & 46,2 & 34,0 & 27,6 & 37,2 & 29,5 \\
\hline Estado civil & & $(0,090)$ & $(0,190)$ & $(0,433)$ & $(0,502)$ & $(0,241)$ \\
\hline Não casada & $233(36,1)$ & 30,0 & 30,9 & 33,9 & 33,5 & 31,1 \\
\hline Casada & $413(63,9)$ & 35,4 & 34,6 & 32,9 & 33,2 & 34,3 \\
\hline Cor da pele & & $(0,373)$ & $(0,205)$ & $(0,027)^{*}$ & $(0,261)$ & $(0,107)$ \\
\hline Branca & $453(70,1)$ & 34,0 & 32,2 & 35,8 & 32,5 & 31,6 \\
\hline Não branca & $192(29,8)$ & 33,5 & 35,9 & 27,6 & 35,4 & 37,0 \\
\hline Escolaridade & & $(0,266)$ & $(0,048)^{*}$ & $(0,712)$ & $(0,991)$ & $(0,028)^{*}$ \\
\hline$\leq 4$ anos & $174(26,9)$ & 30,1 & 31,0 & 32,2 & 33,3 & 39,1 \\
\hline 5 a 6 anos & $164(25,5)$ & 32,3 & 39,0 & 32,3 & 32,9 & 36,6 \\
\hline 7 a 9 anos & $161(24,9)$ & 29,2 & 37,3 & 31,7 & 32,9 & 31,7 \\
\hline$\geq 10$ anos & $145(22,7)$ & 32,4 & 25,5 & 37,2 & 34,5 & 24,1 \\
\hline Renda familiar & & $(0,495)$ & $(0,076)$ & $(0,491)$ & $(0,924)$ & $(0,050)^{*}$ \\
\hline$\leq 2 \mathrm{SM}$ & $185(28,8)$ & 31,4 & 28,6 & 34,1 & 31,9 & 36,8 \\
\hline 2,01 a $3 \mathrm{SM}$ & $135(20,9)$ & 29,6 & 35,6 & 31,1 & 35,6 & 36,3 \\
\hline 3,01 a $5 \mathrm{SM}$ & $172(26,8)$ & 36,6 & 40,1 & 30,2 & 33,1 & 34,9 \\
\hline$\geq 5,01 \mathrm{SM}$ & $151(23,5)$ & 35,8 & 29,1 & 37,7 & 33,1 & 23,8 \\
\hline Estado nutricional & & $(0,038)^{*}$ & $(0,848)$ & $(0,036)^{*}$ & $(0,207)$ & $(0,596)$ \\
\hline Normal & $127(19,6)$ & 30,7 & 35,4 & 42,5 & 38,6 & 37,0 \\
\hline Sobrepeso & $226(35,0)$ & 28,3 & 32,7 & 29,2 & 34,5 & 31,9 \\
\hline Obeso & $293(45,4)$ & 38,6 & 32,8 & 32,4 & 30,0 & 32,8 \\
\hline Estado menopáusico & & $(0,049)^{*}$ & $(0,869)$ & $(0,082)$ & $(0,092)$ & $(0,011)^{*}$ \\
\hline Pré-menopausa & $111(17,8)$ & 24,3 & 32,4 & 36,0 & 26,1 & 35,1 \\
\hline Perimenopausa & $302(46,7)$ & 33,8 & 34,1 & 36,4 & 32,1 & 38,1 \\
\hline Pós-menopausa & $228(35,5)$ & 37,7 & 32,0 & 27,6 & 37,7 & 25,9 \\
\hline
\end{tabular}

PA I: frutas/verduras; PA II: brasileiro; PA III: lanches; PA IV: prudente; PA V: regional; SM: salários mínimos; p-valor: teste Qui-quadrado de Pearson; ${ }^{*}$ p-valor $\leq 0,05$.

do estilo ocidental. Padrões alimentares com características semelhantes também foram encontrados em outros estudos ${ }^{18-21}$.

O padrão tradicional identificado neste estudo foi caracterizado pelo consumo de alimentos tradicionais do brasileiro, sendo feijão, arroz, leite integral, refrigerante/refresco, laranja e manteiga. Apesar de algumas investigações terem relatado um abandono progressivo da dieta tradicional brasileira $^{22,23}$, o padrão tradicional deste estudo foi similar ao de risco de baixo custo encontrado por Alves et al. ${ }^{24}$, em estudo com mulheres adultas da região próxima a desta pesquisa. Além disso, este também foi o padrão tradicional encon- trado por Sichieri ${ }^{25}$ e Sichieri et al. ${ }^{26}$ em estudos realizados no Brasil.

O padrão alimentar frutas e verduras foi associado diretamente com a idade das mulheres, à medida que aumentava a idade crescia o consumo deste padrão. Outros estudos, inclusive no sul do Brasil, já identificaram esta tendência, ou seja, as mulheres adquirem hábitos alimentares mais saudáveis com o aumento da idade ${ }^{18,27-29}$. Este padrão também foi mais consumido pelas mulheres que estavam na pós-menopausa, provavelmente em função desta relação com a idade. As mulheres com obesidade apresentaram maior consumo deste padrão e as com peso normal consumiam 
Tabela 3. Razões de prevalência (RP) para o efeito das variáveis socioedemográficas e do estado nutricional e menopáusico sobre o consumo dos padrões alimentares (PA) I e V de mulheres no climatério em atendimento ambulatorial no Sul do Brasil. ( $\mathrm{N}=646)$

\begin{tabular}{|c|c|c|c|c|}
\hline \multirow{2}{*}{ Variável } & \multicolumn{2}{|c|}{$\begin{array}{c}\text { PA I } \\
\text { (p-valor) } \\
\text { RP (IC95\%) }\end{array}$} & \multicolumn{2}{|c|}{$\begin{array}{c}\text { PA V } \\
\text { (p-valor) } \\
\text { RP }(\text { IC95\%) }\end{array}$} \\
\hline & Bruta & Ajustada & Bruta & Ajustada \\
\hline Idade & $(0,001)^{*}$ & $(0,007)^{*}$ & $(0,053)$ & $(0,643)$ \\
\hline 40 a 45 anos & 1 & 1 & 1 & 1 \\
\hline 46 a 50 anos & $1,01(0,93-1,08)$ & $1,01(0,93-1,09)$ & $0,97(0,90-1,05)$ & $0,99(0,92-1,07)$ \\
\hline 51 a 55 anos & $1,02(0,94-1,11)$ & $1,04(0,95-1,14)$ & $0,93(0,86-1,01)$ & $0,98(0,89-1,07)$ \\
\hline 56 a 65 anos & $1,14(1,05-1,23)$ & $1,15(1,04-1,28)$ & $0,93(0,86-1,01)$ & $1,02(0,92-1,13)$ \\
\hline Estado civil & $(0,167)$ & $(0,108)$ & $(0,428)$ & - \\
\hline Não casada & 1 & 1 & 1 & \\
\hline Casada & $1,04(0,98-1,10)$ & $1,05(0,99-1,11)$ & $1,02(0,97-1,08)$ & \\
\hline Cor da pele & $(0,674)$ & - & $(0,185)$ & $(0,426)$ \\
\hline Branca & 1 & & 1 & 1 \\
\hline Não branca & $0,99(0,93-1,05)$ & & $1,04(0,98-1,10)$ & $1,02(0,96-1,08)$ \\
\hline Escolaridade & $(0,148)$ & $(0,322)$ & $(0,003)^{*}$ & $(0,004)^{*}$ \\
\hline$\leq 4$ anos & 1 & 1 & 1 & 1 \\
\hline 5 a 6 anos & $0,95(0,88-1,02)$ & $0,96(0,89-1,03)$ & $0,98(0,91-1,06)$ & $0,99(0,92-1,07)$ \\
\hline 7 a 9 anos & $0,92(0,86-1,00)$ & $0,94(0,87-1,01)$ & $0,95(0,88-1,02)$ & $0,94(0,86-1,01)$ \\
\hline$\geq 10$ anos & $0,95(0,88-1,03)$ & $0,97(0,90-1,05)$ & $0,90(0,83-0,96)$ & $0,89(0,82-0,97)$ \\
\hline Renda familiar & $(0,229)^{*}$ & - & $(0,016)^{*}$ & $(0,040)^{*}$ \\
\hline$\leq 2 \mathrm{SM}$ & 1 & & 1 & 1 \\
\hline 2,01 a $3 \mathrm{SM}$ & $0,98(0,91-1,06)$ & & $0,99(0,92-1,08)$ & $1,00(0,93-1,08)$ \\
\hline 3,01 a $5 \mathrm{SM}$ & $1,04(0,97-1,12)$ & & $0,99(0,92-1,06)$ & $0,99(0,84-0,98)$ \\
\hline$\geq 5,01 \mathrm{SM}$ & $1,03(0,96-1,11)$ & & $0,90(0,84-0,97)$ & $0,91(0,83-0,97)$ \\
\hline Estado nutricional & $(0,045)^{*}$ & $(0,070)$ & $(0,499)$ & - \\
\hline Normal & 1 & 1 & 1 & \\
\hline Sobrepeso & $0,98(0,91-1,06)$ & $0,98(0,91-1,06)$ & $0,96(0,89-1,04)$ & \\
\hline Obeso & $1,06(0,98-1,14)$ & $1,05(0,97-1,31)$ & $0,97(0,90-1,04)$ & \\
\hline Estado menopáusico & $(0,016)^{*}$ & $(0,771)$ & $(0,021)^{\star}$ & $(0,062)$ \\
\hline Pré-menopausa & 1 & 1 & 1 & 1 \\
\hline Perimenopausa & $1,05(1,00-1,09)$ & $1,04(0,96-1,12)$ & $1,02(0,95-1,10)$ & $1,02(0,94-1,10)$ \\
\hline Pós-menopausa & $1,20(1,10-1,31)$ & $0,98(0,88-1,09)$ & $0,93(0,86-1,01)$ & $0,91(0,82-1,00)$ \\
\hline
\end{tabular}

PA I: frutas/verduras; PA V: regional; SM: salários mínimos; p-valor: regressão de Poisson; ${ }^{\star}$ p-valor $\leq 0,05$.

mais o padrão lanches. Desta forma, levanta-se a hipótese que a maioria das mulheres negligenciam sua alimentação enquanto não sofrem consequências visíveis desta escolha, porém, quando estas se tornam visíveis elas passam a adotar hábitos mais saudáveis.

O padrão alimentar regional apresentou associação inversa com escolaridade e renda familiar, mulheres com menor escolaridade e renda familiar consumiram mais este padrão. Outro estudo também observou que um padrão alimentar de risco, com baixo custo, foi o mais consumido pelas mulheres das classes econômicas C, D e
E, sendo que este padrão se assemelha ao regional do presente estudo ${ }^{24}$.

A relação entre o padrão regional com menor escolaridade e renda pode em parte ser explicada por ser composto por alimentos que têm possibilidade de fabricação caseira, com menor custo e maior tempo de preparo, provavelmente adotado mais pelas donas de casa - como macarrão, batata, aipim, carne suína e bovina - sugerindo que este preparo caseiro ainda faz parte da cultura na região de realização do estudo. Este achado também foi observado em uma pesquisa do IBGE 2008-2009, em que as prepa- 
rações culinárias caseiras ainda fazem parte da alimentação dos brasileiros (cerca de 68,9\% do total de calorias consumidas) ${ }^{30}$. Deve-se levar em consideração também o componente étnico da região, sendo composto por descentes italianos e alemães que são conhecidos pela a importância que dão à culinária caseira. Outra explicação seria a associação entre maior renda e escolaridade e maior adesão a padrões alimentares prudentes evidenciadas por outros estudos ${ }^{28,31}$. No entanto, identificamos que o padrão alimentar II (brasileiro) foi mais consumido pelas mulheres com escolaridade média enquanto o padrão alimentar $\mathrm{V}$ (regional) pelas com baixa escolaridade.

O estado menopáusico revelou diferentes consumos alimentares conforme as fases do climatério. As mulheres na perimenopausa consumiram mais o padrão alimentar V (regional), enquanto que aquelas na pós-menopausa consumiram mais o padrão I (frutas e verduras). Em estudo realizado com mulheres australianas de 18 a 79 anos também foi verificado diferença nos padrões alimentares ingeridos de acordo com o estado menopáusico: o padrão petiscos e álcool e o padrão carne, gordura e alimentos açucarados foi mais consumido pelas mulheres na pré-menopausa, enquanto o padrão de frutas, legumes, verduras e grãos integrais por quem estava na pós-menopausa ${ }^{21}$. Contudo, outros estudos sobre o tema não encontraram diferença na ingestão de alimentos em mulheres na menopausa ${ }^{32,33}$. Assim, as diferenças entre estado menopáusico e padrões alimentares verificado em nosso estudo devem-se possivelmente a outras características como fatores sociodemográficos.

Os padrões alimentares obtidos neste estudo são parte, com algumas exceções, do que é ideal dentre as recomendações do mais recente guia alimentar da população brasileira ${ }^{34}$. Destaca-se, a presença predominante do consumo de alimentos in natura e preparações culinárias. Além disso, os alimentos que compõem os padrões são semelhantes aos encontrados na pesquisa do IBGE 2008-2009 que fundamentou o novo guia: preparações à base de cereais (em especial arroz, e de feijão), carnes de boi ou de porco (carnes vermelhas), carne de frango, leite, raízes e tubérculos (em especial, mandioca e batata), frutas, peixes, legumes e verduras e ovos. Já os produtos prontos para consumo são ingeridos esporadicamente ou em quantidades muito reduzidas, sendo que nos achados deste estudo emergiu somente um padrão com predominância de produtos ultraprocessados, denominado padrão lanches.
Indivíduos que seguem as recomendações do guia alimentar têm um perfil nutricional que atende ou fica muito próximo das recomendações internacionais para a densidade de energia e para o teor de proteína, gordura (vários tipos), açúcar e fibras. Pequenas mudanças no consumo alimentar da população do estudo, incluindo um aumento no consumo de legumes e verduras e uma diminuição no consumo de carnes vermelhas, tornaria o perfil nutricional de sua alimentação praticamente ideal ${ }^{34}$.

Dentre as limitações deste estudo, destaca-se que os achados encontrados devem ser interpretados à luz da possibilidade de viés de causalidade reversa, característica do desenho do estudo transversal utilizado. Isso pode ser observado principalmente quanto ao maior consumo do padrão alimentar frutas e verduras pelas mulheres mais velhas. Ou seja, elas podem ter adquirido hábitos mais saudáveis após a ocorrência de alguma morbidade, como, por exemplo, hipertensão ou obesidade. Outra possível limitação do estudo é a possibilidade de viés de informação quanto ao relato de consumo dos alimentos. Para minimizar esse viés, todos os itens alimentares do QFA utilizado foram lidos e, sistematicamente, se enfatizou que se tratava do consumo do alimento no último ano. Outra limitação refere-se ao uso do QFA, ao qual pode limitar ou extinguir escolhas étnicas ou alimentos específicos ao colocá-los em um grupo maior ${ }^{32}$. Por esta razão, tomou-se o cuidado para formar grupos de alimentos que são significativos, tanto para o estudo populacional quanto para a questão de pesquisa. Porém, ao adaptar o QFA em nosso estudo, passou despercebido um alimento de grande consumo na região, a polenta. Esse desvio pode ter sido reduzido pelo surgimento de um padrão alimentar regional, onde provavelmente este alimento se encaixaria, no entanto, poderia elevar o consumo deste padrão. Por fim, a identificação de padrões alimentares por meio da análise de componentes principais merece atenção. Essa abordagem envolve algumas decisões, como a escolha dos itens alimentares a serem incluídos no QFA, o agrupamento dos itens alimentares, o número de padrões a ser extraído, o método de rotação, além da denominação dos padrões alimentares.

Este estudo pode ser considerado relevante, por ser o primeiro a identificar os padrões alimentares em mulheres da região da serra gaúcha, bem como a associação com os estados menopáusicos. Ressalta-se a importância da fase de vida estudada, o climatério, visto que durante 
a transição da pré-menopausa para a pós-menopausa as mulheres apresentam elevados índices de sobrepeso/obesidade e pouco se conhece sobre a alimentação nesta fase. Além disso, os resultados deste estudo proporcionam informações que poderão ser utilizadas em ações de promoção da saúde e de assistência às mulheres no climatério, no âmbito dos serviços públicos de saúde, considerando as possíveis representações de insegurança diante os sintomas de natureza física e psicológica da menopausa ${ }^{35}$. Assim, foram

\section{Colaboradores}

M Hoffmann, KG Mendes e MTA Olinto participaram da concepção, delineamento e na análise e interpretação dos dados. R Canuto, AS Garcez, H Theodoro e AD Rodrigues participaram da redação e revisão crítica do artigo. Todos os autores aprovaram a versão final do manuscrito.

\section{Agradecimentos}

Ao Conselho Nacional de Desenvolvimento Científico e Tecnológico, à Fundação de Amparo à Pesquisa do Estado do Rio Grande do Sul e ao Programa de Apoio a alunos de Pós-Graduação da Unisinos-Promestre, pelo financiamento. identificados cinco padrões que descreveram o consumo alimentar da população estudada, sendo este semelhante ao indicado como ideal para a população brasileira. Além disso, mostraramse consistentes com uma expectativa a priori de padrões, principalmente com a identificação de um típico da região estudada. Nossos achados indicam que o padrão alimentar das mulheres no climatério possui influência significativa da idade, escolaridade e renda, mas não do estado menopáusico.

\section{Referências}

1. Organización Mundial de la Salud (OMS). Investigaciones sobre la menopausa en los anos noventa: informe de un grupo cientifico de la OMS. Ginebra: OMS; 1996. (OMS, Serie de informes técnicos, nº 866.

2. Santos EF, Marcellini PS, Melo MG, Almeida ML. Avaliação do consumo alimentar e do perfil lipídico de mulheres na menopausa. Rev Bras Cardiologia 2008; 40(4):267-271.

3. Biel RK, Friedenreich CM, Csizmadi I, Robson PJ, McLaren L, Faris P, Courneya KS, Magliocco AM, Cook LS. Case-control study of dietary patterns and endometrial cancer risk. Nutr Cancer 2011; 63(5):673-686.

4. Esmaillzadeh A, Azadbakht L. Food intake patterns may explain the high prevalence of cardiovascular risk factors among Iranian women. J Nutr 2008; 138(8): 1469-1475.

5. Esmaillzadeh A, Azadbakht L. Major dietary patterns in relation to general obesity and central adiposity among Iranian women. J Nutr 2008; 138(2):358-363.

6. Fairweather-Tait SJ, Skinner J, Guile GR, Cassidy A, Spector TD, MacGregor AJ. Diet and bone mineral density study in postmenopausal women from the TwinsUK registry shows a negative association with a traditional English dietary pattern and a positive association with wine. Am J Clin Nutr 2011; 94(5):13711375.

7. Lopez EP, Rice C, Weddle DO, Rahill GJ. The relationship among cardiovascular risk factors, diet patterns, alcohol consumption, and ethnicity among women aged 50 years and older. J Am Diet Assoc 2008; 108(2):248-256.

8. Martinazzo J, Zemolin GP, Spinelli RB, Zanardo VPS, Ceni GC. Avaliação nutricional de mulheres no climatério atendidas em ambulatório de nutrição no norte do Rio Grande do Sul, Brasil. Cien Saude Colet 2013; 18(11):3349-3356.

9. Hu FB. Dietary pattern analysis: a new direction in nutritional epidemiology. Curr Opin Lipidol 2002; 13(1): 3-9. 
10. Cottet V, Touvier M, Fournier A, Touillaud MS, Lafay L, Clavel-Chapelon F, Boutron-Ruault MC. Postmenopausal breast cancer risk and dietary patterns in the E3N-EPIC prospective cohort study. Am J Epidemiol 2009; 170(10):1257-1267.

11. Fung TT, Schulze M, Manson JE, Willett WC, Hu FB. Dietary patterns, meat intake, and the risk of type 2 diabetes in women. Arch Intern Med 2004; 164(20):22352240.

12. Rodrigues AD, Theodoro H, Mendes KG, Paniz VM, de Lorenzi D, Anselmo Olinto MT. Factors associated with metabolic syndrome in climacteric women of southern Brazil. Climacteric 2013; 16(1):96-103.

13. Theodoro H, Rodrigues AD, Mendes KG, Liane RH, Paniz VM, Olinto MT. Reproductive characteristics and obesity in middle-aged women seen at an outpatient clinic in southern Brazil. Menopause 2012; 19(9):1022-1028.

14. World Health Organization (WHO). Physical status: the use and interpretation of anthropometry. Geneva: WHO; 1995. (Report of the WHO Expert Committee. World Health Organ Tech Rep Ser).

15. World Health Organization (WHO). Obesity: Preventing and managing the global epidemic - Report of a WHO consultation on obesity. Geneva: WHO; 2000. (WHO Technical Report Series, no 894).

16. Anjos LA, Wahrlich V, Vasconcellos MTL, de Souza DR, Olinto MTA, Waissmann W, Henn RL, Rossato SL, Lourenço AE, Bressan AW. Development of a food frequency questionnaire in a probabilistic sample of adults from Niterói, Rio de Janeiro, Brazil. Cad Saude Publica 2010; 26(11):2196-2204.

17. Hair JF. Análise multivariada de dados. Porto Alegre: Artmed; 2005.

18. Cho YA, Kim J, Cho ER, Shin A. Dietary patterns and the prevalence of metabolic syndrome in Korean women. Nutr Metab Cardiovasc Dis 2011; 21(11):893-900.

19. Fung TT, Hu FB, Barbieri RL, Willett WC, Hankinson SE. Dietary patterns, the Alternate Healthy Eating Index and plasma sex hormone concentrations in postmenopausal women. Int J Cancer 2007; 121(4):803-809.

20. Fung TT, Willett WC, Stampfer MJ, Manson JE, Hu FB. Dietary patterns and the risk of coronary heart disease in women. Arch Intern Med 2001; 161(15):1857-1862.

21. Kolahdooz F, Ibiebele TI, van der Pols JC, Webb PM. Dietary patterns and ovarian cancer risk. Am J Clin Nutr 2009; 89(1):297-304.

22. Levy-Costa RB, Sichieri R, Pontes Ndos S, Monteiro CA. Disponibilidade domiciliar de alimentos no Brasil: distribuição e evolução (1974-2003). Rev Saude Publica 2005; 39(4):530-540.

23. Pereira RA, Andrade RG, Sichieri R. Mudanças no consumo alimentar de mulheres do Município do Rio de Janeiro, Brasil, 1995-2005. Cad Saude Publica 2009; 25(11):2419-2432.
24. Alves AL, Olinto MT, Costa JS, Bairros FS, Balbinotti MA. Padrões alimentares de mulheres adultas residentes em área urbana no sul do Brasil. Rev Saude Publica 2006; 40(5):865-873.

25. Sichieri R. Dietary patterns and their associations with obesity in the Brazilian city of Rio de Janeiro. Obes Res 2002; 10(1):42-48

26. Sichieri R, Castro JF, Moura AS. Fatores associados ao padrão de consumo alimentar da população brasileira urbana. Cad Saude Publica 2003; 19(Supl. 1):S47-53.

27. Canuto R, Camey S, Gigante DP, Menezes AM, Olinto MT. Focused Principal Component Analysis: a graphical method for exploring dietary patterns. Cad Saude Publica 2010; 26(11):2149-2156.

28. Lenz A, Olinto MT, Dias-da-Costa JS, Alves AL, Balbinotti M, Pattussi MP, Bassani DG. Socioeconomic, demographic and lifestyle factors associated with dietary patterns of women living in Southern Brazil. Cad Saude Publica 2009; 25(6):1297-1306.

29. Neumann AI, Martins IS, Marcopito LF, Araujo EA. Padrões alimentares associados a fatores de risco para doenças cardiovasculares entre residentes de um município brasileiro. Rev Panam Salud Publica 2007; 22(5): 329-339.

30. Instituto Brasileiro de Geografia e Estatística (IBGE). Pesquisa de orçamentos familiares 2008-2009. Brasília: IBGE; 2010 .

31. Kant AK. Dietary patterns and health outcomes. J Am Diet Assoc 2004; 104(4):615-635.

32. Macdonald HM, New SA, Reid DM. Longitudinal changes in dietary intake in Scottish women around the menopause: changes in dietary pattern result in minor changes in nutrient intake. Public Health Nutr 2007; 8(04):409-416.

33. Riesco E, Tessier S, Perusse F, Turgeon S, Tremblay A, Weisnagel J, Doré J, Mauriège P. Impact of walking on eating behaviors and quality of life of premenopausal and early postmenopausal obese women. Menopause 2010; 17(3):529-538

34. Brasil. Ministério da Saúde (MS). Guia alimentar para a população brasileira. Brasília: MS; 2014.

35. Mendonça EAP. Representações médicas e de gênero na promoção da saúde no climatério/menopausa. Cien Saude Colet 2004; 9(3):751-762.

Artigo apresentado em 18/06/2014

Aprovado em 09/10/2014

Versão final apresentada em 11/10/2014 